\title{
Esto no es una biografía: Fuguet sobre Caicedo
}

\author{
This is not a biography: \\ Fuguet on Caicedo
}

\author{
PAOLA PIACENZA \\ ${ }^{a}$ Universidad Nacional de Rosario, Argentina. \\ Correo electrónico: ppiacenza@gmail.com
}

El propósito de este trabajo es discutir los presupuestos de la renuncia de Alberto Fuguet a escribir una biografía de Andrés Caicedo que pueden leerse no solo en la caracterización de Mi cuerpo es una celda (2008) como "una autobiografía" y un "documental narrado en primera persona”, sino en sus reflexiones acerca del género en el "making of" de la obra y en las notas y apuntes para un ensayo sobre Caicedo escritas cuatro años más tarde.

Las decisiones del biógrafo — que se declara "director y montajista" de la obra— ponen en juego algunos problemas constitutivos de la biografía como la tensión entre documentación y artificio; realidad y ficción; vida y obra, pero también introducen otros singulares; consecuencia de la naturaleza del vínculo que construye Fuguet con Caicedo a través de la lectura y la investigación, pero, sobre todo, por su pasión compartida por el cine.

Palabras clave: biografía, autobiografía, Fuguet, Caicedo, cine.

The purpose of this paper is to analyze the implications of Alberto Fuguet's reluctance to write a biography of Andres Caicedo, which can be gathered from the author's characterization of Mi Cuerpo es una Celda (2008) as an "autobiography" and a documentary narrated in the first person, as well as in the reflection on the genre in "the making of" of the mentioned work, and in the notes for an essay about Caicedo written four years later.

The decisions of the biographer — who assumes the role of a director and editor of the bookrepresent for us an opportunity to discuss certain critical concerns about the biography as a genre such as the tension between factuality and fiction; reality and text; life and work. In addition, they lead to another specific inquiries related to the particular case of this text which emerge from Fuguet's research, his confessed passion for Caicedo's literature and, above all, their shared love of cinema and movies.

Key words: biography, autobiography, Fuguet, Caicedo, cinema. 


\section{El Proceso}

En el año 2008, Alberto Fuguet publica Mi cuerpo es una celda. El libro tiene como subtítulo "Una autobiografía" y el autor se presenta a sí mismo, en la portada, como responsable de la "dirección y montaje" de la obra. Desde el primer contacto, el lector de Mi cuerpo es una celda tiene que resolver las contradicciones que introduce la información del paratexto editorial: por un lado, en relación con el "horizonte de expectativa" creado por los géneros nombrados y aludidos (autobiografía, cine); por el otro, por la difícil atribución referencial de los nombres propios y las formas gramaticales en juego (las referencias al chileno Alberto Fuguet y al colombiano Andrés Caicedo; el pronombre que atribuye la posesión de un cuerpo a un sujeto que no es el autor; el artículo que indica la indeterminación del texto autobiográfico).

Desde el inicio, la obra se instala en la zona que media entre el problema gramatical y el de la identidad (Lejeune 49) en la literatura del yo, para producir ("hacer" es el verbo que elige Fuguet) la escritura de una vida (la de Caicedo) por fuera del registro de la propiedad que distingue la vida propia de la ajena; más allá del valor indicial de los pronombres y, como veremos a continuación, con prescindencia de las exigencias lógicas que supone la diégesis narrativa. En otras palabras, reniega de la mayoría de las convenciones a las que se ha apelado para distinguir las fronteras que definen a la biografía entre los distintos textos que configuran el campo de las escrituras de vida. Así, si el subtítulo de la tapa alude a "una autobiografía", la edición culmina con un capítulo que se denomina "Cómo se hizo este libro" (Fuguet y Caicedo 2561) — y que dice ser el "making of" de la obraen donde la idea autobiográfica se retoma, pero ahora en función de la posibilidad de escribir una "biografía" de Caicedo y en una sección cuya caracterización elude la propia idea de representación. En este capítulo final, las variaciones formales y experimentaciones enunciativas, que se reconocen desde el primer momento, se revelan como alternativas a la escritura biográfica de la que el autor desiste, al menos como propósito. Nuestro interés será, entonces, comprender qué se entiende, en este contexto, por biografía y qué posibilidades depara esa renuncia como premisa.

La "biografía" que Fuguet rechaza para contar la vida de Caicedo es la que se define como género referencial —asociado a un material documental — y como especie narrativa, es decir, el relato de la vida de un personaje (protagonista) que, en su forma canónica, ejerce un narrador en tercera persona y en posición heterodiegética. A Fuguet las biografías, especialmente las muy "investigadas", le parecen falsas tanto desde una perspectiva moral (cuestiona la verdad por adecuación a las fuentes) como discursiva, por la coherencia que impone la narración al curso inapresable de una vida. En principio, la renuncia parece obedecer a una razón práctica. Una vez que definió su lugar de autor en relación con la obra en el rol de "montajista", dice que se encontró con mucho material, con un director-

\footnotetext{
${ }^{1}$ En la referencia de las citas de Mi cuerpo es una celda respeté la coautoría que supone el copyright compartido por Fuguet y Caicedo. Un indicador más de la ambigüedad propuesta por la obra.
} 
guionista que ya no estaba y con unos productores "que sólo querían que se respetara la visión del autor" (Fuguet y Caicedo 257) y eso era imposible de hacer "sin poder hablar con él". Como Fuguet ya no puede hablar con Caicedo (el colombiano se suicida veinte años antes, en 1977), promete ser fiel al archivo, pero rápidamente concluye "Cuán ficticias pueden ser las biografías, sobre todo las más investigadas. No es que cada fuente mienta, es simplemente que no estuvieron alli” (258). Después de regresar de Cali y advertir que cada entrevistado le había contado algo distinto y contradictorio sobre el escritor, concluye que una "autobiografía" se le presenta como una opción pertinente a la escritura de una biografía: si "necesariamente" (toda autobiografía) "tiene que ser escrita por la persona que ha vivido esa vida" (257), este era, literalmente, el caso del libro. Caicedo es quien dice "yo" en las cartas, notas, listas y apuntes en cuadernos que componen el volumen.

Decíamos que el otro motivo de la renuncia a la biografía es el rechazo del verosímil que Pierre Bourdieu llamó "ilusión biográfica” (Bourdieu 1997). A este respecto, cuatro años después de publicar la obra que nos ocupa, en el 2012, Fuguet escribe el artículo "25 años de soledad. Notas y apuntes para un ensayo acerca de Andrés Caicedo, luego de terminar y publicar Mi cuerpo es una celda, su 'autobiografía'" (Fuguet Cinépata 203) y en uno de los primeros apuntes vuelve a pensar sobre las contradicciones del oficio del biógrafo atrapado entre la materia y la interpretación. El texto forma parte de Cinépata. Una bitácora (2012) un libro en el que Fuguet reúne citas, apuntes, críticas, artículos, ensayos y entradas diarísticas a propósito de su relación "enfermiza” con el cine. En este apunte consigna nuevamente su rechazo por el carácter inauténtico de las biografías, pero esta vez a partir de otro argumento: la aspiración a la totalidad en la que descansa la credibilidad del relato. Por ejemplo, a modo de "spin off" del "making of" se refiere a un material que no incluyó en la recopilación de Mi cuerpo es una celda. Se trata de una carta que escribió el exmarido de una de las hermanas de Caicedo, psiquiatra, en la que no solo predice el inevitable suicidio de Caicedo, sino que realiza un "diagnóstico" de toda la familia. Fuguet dice haber sido consciente de la importancia de esa carta para la comprensión de las circunstancias de la vida de Caicedo pero, no obstante, explica las razones de la omisión:

No la usé porque parte de la tragedia de la autobiografía es que se parece a la vida; es decir, la persona que cuenta no siempre sabe todo de sí mismo, no siempre conoce los factores externos ni sabe lo que le va a suceder. Por eso me parece un género más digno, más fascinante, más verdadero que las biografías (Fuguet Cinépata 207).

El volumen, al mismo tiempo que se dispersa en apuntes, textos breves similares a las entradas de un diario, muchas cartas y una biografía apócrifa, se reconoce como unidad también en una sucesión de páginas que, a partir del índice, remedan la continuidad que asegura la existencia de una vida: "maldita cosa, después de otra cosa", de acuerdo con las palabras de Lord Chesterfield, según la cita de memoria Borges en una conferencia en Oxford. El "montaje" de Fuguet ensambla los materiales en tres momentos progresivos: “Cambiando/encontrando la voz" (1966-1972), "Cali calabozo" (1973-1976) y "El espiral 
descendente" (1976-1977). El índice se organiza, de este modo, sobre dos líneas que se superponen y resignifican entre sí: la de la cronología histórica y la de los "períodos" (o episodios significativos). De este modo, estas etapas restablecen no solo el orden secuencial de los días y los papeles de Caicedo sino el carácter "argumental" de una "historia de vida": "(...) ojalá pudiera leerse como una novela, como una novela de no ficción" — concluye Fuguet en el "Making of" - "Lo importante era que todo ese material disperso, casi siempre reiterativo, pudiera leerse como una pieza orgánica" (Fuguet y Caicedo 258). Los sentidos de la edición escriben la novela, finalmente, contra las aspiraciones de neutralidad.

Es cierto que podría objetarse el hecho de que la serie temporal no es cronológica o progresiva porque está precedida por la carta en la que — dos años antes de morirCaicedo le pide a su madre que comprenda su muerte, sin embargo, en la página opuesta al texto, la foto infantil del escritor le confiere entidad y unidad a una vida que "se mantiene [mantuvo] en un hilo" (Fuguet y Caicedo 201), de acuerdo con su propia definición. La foto del nińo intenta, como toda imagen, negar la ausencia y, a modo de los retratos que ilustran las construcciones funerarias, documenta el carácter necrológico (y necrofílico) de toda biografía: "Adiós" no es solo el saludo de despedida de la última carta recopilada en el libro; es también la última palabra de Mi cuerpo es una celda.

Contra los prejuicios de la ficción biográfica, Fuguet recurre a los prejuicios de la ficción documental: escribe una "historia basada en hechos reales". La coartada, sin embargo, se la proveen las convenciones del propio género biográfico. En el mismo momento en que renuncia a contar, delega la responsabilidad de la forma en la selección. Aquello que, en palabras de Julia Musitano, “... pretende consignarse como una falta (y) constituye, en realidad, la esencia del género ... " (Musitano 169). Fuguet insiste en señalar que el libro no solo fue "montado" sino "editado": "Algunas cartas fueron reducidas. Otras, de la misma fecha, se fusionaron. Aquellos escritos que aparecen como apuntes o posts o anotaciones en un diario de vida son un invento mío a partir de muchas frases de Andrés que aparecían en largas cartas centradas en temas ni cinéfilos ni personales" (Fuguet y Caicedo 264). Contra las intenciones de Fuguet, el montaje opera en la conversión de la "autobiografía" en "biografía" y no podría serlo de otro modo. En primer lugar, porque el montaje, a diferencia, de un collage, ordena y empalma los materiales según una idea previa y un ritmo determinado. En segundo lugar, y acaso sea la razón más importante, porque se sabe que las formas "contemporáneas" de la biografía (las de las primeras décadas del siglo $\mathrm{XX}$ ) y las del cine se han nutrido mutuamente. En su artículo, "The newness of the new biography", Laura Marcus (2002) señala que "la nueva biografía ha recibido la influencia de las técnicas cinematográficas del detalle, la atención sobre el gesto y el close up y por las subversiones del tiempo lineal y la cronología, mientras que el cine ha tomado prestado de la biografía la trayectoria de una vida como un legítimo asunto cinematográfico y un principio estructural" (Marcus 215) ${ }^{2}$. En este sentido, si el montaje reenvía a la escritura biográfica, la tarea de "dirección" que Fuguet asume desde la portada del libro no hace sino confirmarlo.

\footnotetext{
${ }^{2}$ La traducción del inglés al español es mía.
} 
"Lo de director tuvo que ver en darle un tono" (257) — dice Fuguet— y efectivamente, si el "proceso" al que aludía el autor en relación con el objeto de su obra se ha jugado en relación con el montaje; el "lazo" —entre el biógrafo y el biografiado— se revela en ese tono.

\section{El LaZo}

Hasta aquí las razones del autor; el programa de la escritura de Mi cuerpo es una celda en sus propios términos. Sin embargo, si le creemos al propio Fuguet, no hay nada más falso que aquello que se presenta como evidente y ostenta una razonable sensatez. Es claro que la explicación da cuenta del proceso de producción de la obra, pero no dice nada sobre los oficios del lazo del biógrafo que se resiste a serlo.

"Proceso" y "lazo" son los dos conceptos a los que apela Fuguet para caracterizar su posición como director y montajista y que resultan imprescindibles para conjeturar sobre los efectos de la biografía que no quiso ser en esta obra. Dice Fuguet: "Quizás algunos hayan notado que el libro que acaban de leer posee el subtítulo de "autobiografía". Otros, por su parte, quizás se fijaron en que mi nombre está asociado a las labores de dirigir y montar este libro. Un libro, claro está, se edita, se revisa, se pule. No se dirige, no hay montaje, ni se utiliza Final Cut Pro. Pero quizás éste sí se montó. No se me ocurre otra manera de entender mi proceso y mi lazo con Mi cuerpo es una celda" (Fuguet Caicedo 257). Parece que es a través del proceso y la tarea del montaje que la biografía de Caicedo se escribe por fuera de las limitaciones que incomodan al autor. El armado descubre la trama que construye los hechos y que la narración biográfica tradicional oculta y, lo que es más importante, vuelve posible el lazo; porque apareja el tiempo y el interés del biógrafo con el del biografiado.

¿Hay acaso una mejor definición del texto biográfico que una escritura que se produce en la alteridad entre la vida propia y la ajena? En este sentido, resulta significativo que entre sus intenciones Fuguet también diga que quiso "hacer algo acerca de Caicedo" y que fuera, al mismo tiempo, un "proyecto" propio, "ligado a mi (su) propio universo/ planeta" (Fuguet Caicedo 257). Ante la pregunta acerca de cómo contar la vida de Caicedo, lo que está claro es que Fuguet renuncia, en primer lugar, a contar: elige callar — dejar hablar a Caicedo-y mostrarlo: "hacer algo".

Sabemos de boca del propio Fuguet que durante su investigación entre Cali y Bogotá recogió centenares de fotocopias de materiales escritos por Caicedo. Sabemos, también, que un año antes (en 2007), la editorial Norma publicó El cuento de mi vida: memorias inéditas, otra selección de papeles de Caicedo, reunidos esta vez bajo el rótulo de "memorias". Parece entonces que nadie quiere escribir la biografía de Caicedo: "autobiografía", "memoria"; en todos los casos se prefiere que Caicedo siga hablando por sí mismo en la poligrafía de sus

\footnotetext{
${ }^{3}$ Es importante señalar que los lugares de reenvío entre las vidas de Fuguet y Caicedo exceden los aquí trabajados estrictamente en función de Mi cuerpo es una celda (entre otros aspectos, los referidos a la disidencia de género, el lugar de sus obras respecto de las posiciones canónicas en sus respectivas literaturas nacionales, etc.).
} 
cuadernos. Si es cierto que "el biógrafo se sienta a escribir una vida ... para retratar la relación entre obra y vida" (Serra Bradford 9). Mi cuerpo es una celda encuentra su destino biográfico en el mismo camino que toma para huir de él porque, en un probable documental sobre Caicedo y su literatura (un "documental" es otra de las definiciones genéricas que ensaya Fuguet para este libro), "obra y vida" caben en la misma toma.

"Dejo algo de obra y muero tranquilo" es probablemente una de las frases más citadas del escritor adolescente. La frase pertenece a una de las dos cartas que escribe el día de su muerte; la que dirige a Patricia Restrepo, quien había sido su pareja hasta poco antes. En este caso la obra retrata al artista. No hay vida de Caicedo por fuera de esa "mala fe de una vida que figura la vida" (256), como definió Maurice Blanchot a la escritura. Caicedo duda de su condición de viviente en la misma medida en que confía en la potencia de su obra. Su condición de "escritor muerto" lo llevará a componer obsesivamente su vida como si fuera parte de su obra. Atento al vértigo del calendario, se autoimpone, desde los diez años, rutinas de lectura, de escritura de ficción, de cartas, de reseñas literarias y cinematográficas; jornadas diarias en las que ve siete u ocho películas. El registro de esos años de formación va conformando una especie de diario que archiva en "folders" y cuadernos "argollados".

En aquellos textos no hay ninguna otra perspectiva de futuro que no sea la construcción de una obra póstuma. No parece casual el hecho de que se suicide (finalmente; había tenido por lo menos otros dos intentos) el mismo día en el que se publica su novela ¿Que viva la música!: la muerte cedía paso, de este modo, a la existencia de la obra. Esta composición de la vida como obra se vuelve evidente en el hecho de que todo comentario biográfico sobre Caicedo incluye casi obligatoriamente la referencia conjunta a su precocidad, disciplina de trabajo y suicidio como si se trataran de tres actos de una trama cuidadosamente planificada. En uno de los tantos textos sobre cine reunidos por Fuguet, Caicedo reflexiona en la conclusión que "... el arte es más organizado que la vida, que si en el arte hay confusión y pena, cuando la transmite es para la diversión" (Caicedo 106). El completo cuento de su vida parece contarse, más precisamente, in media res: la producción frenética pero metódica de relatos, una única novela publicada en vida y reescrita durante cinco años, cientos de reseñas y guiones cinematográficos, obras teatrales propias y adaptaciones, la edición de dos revistas de crítica cinematográfica, diversas puestas en escena de obras propias y ajenas, ocupan vertiginosamente el relato apretado de los "múltiples quehaceres" en los pocos años que median desde su llegada al mundo hasta el día del suicidio. En todos los textos el tiempo de adolescencia es la única temporalidad de su vida con excepción de una referencia mínima y nostálgica a una infancia idílica que antes que un tiempo vital se convierte en el origen de esa reserva moral que lo lleva a horrorizarse ante la posibilidad de "volverse adulto".

"Caicedo se escribía constantemente a sí mismo", dice también Alberto Fuguet, pero unos años antes en el prólogo a la edición colombiana de ¡Que viva la música! (Fuguet "Prólogo" 5) La frase implica no solo el hecho banal de que puedan encontrarse trazos que vinculan a sus personajes e historias con la vida del autor prácticamente en todos sus libros. Antes bien, se produce una singular paradoja entre lo vivido (la supuesta "materia" de la experiencia autobiográfica) y lo vivible (los sentidos posibles — como los negados- por la 
lengua de la obra) por los que pareciera que Caicedo "vivía algunos asuntos de la vida real para poder escribirlos y reflexionar sobre ellos. La vida, para Caicedo, era preferible escrita". Así lo resume su amigo Sandro Romero Rey en la contratapa de la edición de El cuento de mi vida (2007): "Da la impresión de que su autor quería ser una especie de aciago demiurgo al que le estorbaba el ritmo banal de la existencia, pero al que estimulaba a más no poder su traducción en signos escritos".

Si la forma canónica de la narración biográfica se resume en la fórmula "de la cuna a la tumba" (from cradle to grave), la biografía de Caicedo debería salvar el obstáculo de una vida breve: Caicedo ya "ha muerto" para cuando sus días se empeñan en sucederse con la fatalidad del calendario. A los veintidós ańos escribe, en una carta a Patricia, el 30 de julio de 1975: "ya soy lo que he debido o podido ser, de allí no paso y las mejores experiencias (las peores) ya me formaron (me deformaron) ..." (Fuguet Caicedo 174). El tiempo de esta vida contradice las representaciones modernas de la idea de formación: no hay crecimiento, teleología, tampoco aprendizaje. “¿Cuánto hace que no soy nadie? ¿Cuánto hace que soy persona grande?” (181), se cuestiona en otro de los textos editados por Fuguet. En este sentido, la línea cronológica que traza el índice que Fuguet arma para Mi cuerpo es una celda constituye no solo la mayor traición a la pretendida fidelidad documental sino el grado más alto de su intervención biográfica. El índice reproduce las expectativas tradicionales de un relato que dé cuenta de cómo se llegó a ser el que fue mientras que el "contenido" (digámoslo rápidamente de este modo; "ese cuerpo sin órganos" que Caicedo construye) se orienta en la dirección contraria: "un día menos" (y no un día "más") es la temporalidad de la vida escrita por Caicedo.

¿Cómo contar, entonces, la experiencia de una vida que solo acontece en y por la escritura? ¿Cómo escribir la historia de un cuerpo que se sustrae a su condición de "viviente" y de una voz que dice "estoy muerto" (Fuguet Caicedo 206)" sin pretensión de ser metáfora o hipérbole? Philip Holden (2014), en su ensayo, "Literary biography as a critical form" plantea que la biografía constituye un modo de ejercicio crítico que, a diferencia de otros discursos propios de la escritura académica, recurre a la narración. Y puntualiza: "En particular, la biografía literaria tiene el potencial de formular nuevas preguntas sobre las relaciones de un lector con los textos biográficos y literarios de un autor, y de ese modo generar una reflexión en torno a las relaciones entre lectura y subjetividad" $(918)^{4}$. Para Holden, esta posibilidad probablemente haya tenido lugar a partir de que Philippe Lejeune, en 1975, en "El pacto autobiográfico", desplazó el interés de la discusión sobre la autobiografía de la pregunta acerca de las relaciones entre el texto y la vida a una interrogación acerca del tipo de lectura que engendra.

Efectivamente, Fuguet realiza un ejercicio autobiográfico de escritura/lectura crítica (que no hubiera sorprendido a Oscar Wilde) de los textos del escritor sobre el que no duda en expresar su fascinación y esta es la otra dimensión autobiográfica ineludible de la obra. Tal vez no sea casual que solo la portada interna de la edición asigne a Caicedo la autoría de la autobiografía mediante la preposición "por" y esta atribución esté ausente, sin embargo,

\footnotetext{
${ }^{4}$ La traducción del inglés al español es mía.
} 
en la cubierta. $\mathrm{Si}$, en la biografía tradicional, la lectura del biógrafo se escucha en la voz del narrador, aquí se advierte, como ya hemos seńalado, en la composición y el tono — de la edición y de los textos propios que suma a los de Caicedo- pero de hecho está presente desde el inicio, en el fundamento de la propia vocación de restituir la ausencia del escritor - la oportunidad que perdió de ser su amigo; la frustración por haberlo conocido "tarde"a partir de la intervención sobre sus textos y la insistente explicación negativa de los motivos que lo llevaron a prescindir de la biografía como forma para esta obra 5 .

Por cierto, no es la primera vez que Fuguet escribe sobre fantasmas. Prácticamente en simultaneidad con la escritura de Andrés Caicedo. Mi cuerpo es una celda, Fuguet se encuentra abocado a la investigación que daría origen a Missing (una investigación), otra biografía que reniega de su condición, en 2009. Missing presenta más de un punto de contacto con el texto sobre Caicedo. En primer lugar, la indecisión genérica: el subtítulo indica entre paréntesis "(una investigación)" como "crónica", en otras como "entrevista"; "novela de no ficción" y el capítulo que se atribuye a la voz del personaje biografiado -su tío Carlos- es un "eco" de su "pensamiento" (se titula "The Echoes of his Mind"). A pesar de que se diga que ahí "Carlos talks" y se escriba en primera persona, el texto está escanciado en versos ${ }^{7}$ y es una especie de paráfrasis de sus declaraciones en las entrevistas que se consignan unas páginas antes como "fuentes". No obstante, lo más importante, constituye —nuevamente- el hecho de que el propósito manifiesto de la "investigación" es contar la vida de un hombre cuya anécdota principal es una ausencia que se vuelve texto: mientras dura su autoexilio, el tío es "tema" (Fuguet Missing 24) de la conversación (y del silencio) familiar, "mito" (26) y el objeto de una "conversación

${ }^{5}$ Estos desplazamientos en el texto que escribe Fuguet - tanto el que se orienta en dirección al biógrafo (antes que al biografiado) como a la escritura, antes que a la "vida" - pueden reconocerse en las dos biografías de Caicedo que se asumen como tales y que tienen como autores, a su amigo, el cineasta Luis Ospina y, paradójicamente, al propio Caicedo.

Luis Ospina es el autor de "Unos pocos y buenos amigos" (1986), un documental que Fuguet define como "la biografía oral y visual de un fantasma. De alguien que ya no está"; un filme modesto que, según Fuguet prueba que "hay amistades y pérdidas que marcan" aunque no todo el mundo tiene la suerte de "tener un amigo como Luis Ospina”. La otra, la segunda biografía, lleva al paroxismo el problema de la representación biográfica. Se trata de la biografía publicada en la contratapa de la primera edición de los cuentos de El atravesado (1975) y que Fuguet incluye en el "Bonus Track" de Mi cuerpo es una celda. Caicedo se la había pedido al escritor de Barranquilla Jaime Manrique Ardila pero finalmente no pudo hacerlo por lo que Caicedo escribió su propia biografía pero con la firma de Manrique Ardila. En ella, Caicedo resume su trayectoria de vida en tercera persona y desde el futuro anticipado de la desaparición de sí mismo. Después de comentar de qué van los cuentos presentados, concluye ante la amarga evidencia de que han quedado sin respuesta "la cantidad de preguntas que teníamos para hacerle" "ahora después de "su trágica defenestración y descorazonamiento" (Fuguet Caicedo 296).

${ }^{6}$ Fuguet recurre a una estrategia en común en Mi cuerpo es una celda, Missing y Cinépata: la enunciación de un título seguida de una caracterización genérica que antes que resultar explicativa constituye una designación polémica o una complejización de las expectativas de lectura.

${ }^{7}$ En una entrevista con Martín Pérez, publicada en Radar Libros, el 6 de noviembre de 2010, Fuguet define al texto en verso como "una larga canción". 
infernal" (26). Una vez que lo encuentre, tomará el cuerpo de una serie de testimonios: un correo electrónico; el registro de las dos entrevistas desgrabadas. Como en el caso de Caicedo, el tío Carlos Fuguet es la alteridad necesaria para que el escritor revise, a partir de la crónica de la investigación, su propia historia de obsesión con el personaje, en la que el cine - las películas - vuelve a tener un papel ineludible como tercero incluido. El cine cuenta la vida de Carlos desde el título mismo - Fuguet dice que fue él quien lo invitó a ver la mítica película de Costa Gravas en 1982 - y está presente en la evocación de ciertos escenarios (Las Vegas), la construcción de otros personajes (el primo que aspira a ser director) y hasta cierta estética que presenta al protagonista en el imaginario cinematográfico estadounidense de la guerra de Vietnam. Sin embargo, lo más importante, es la percepción de estar asistiendo a la proyección de una road movie que, en su devenir, remueve los sedimentos de las narrativas sobre la búsqueda del padre y del tiempo de aprendizaje. La mediación del cine (tema, estética, géneros) reúne de este modo la experiencia biográfica y la autobiográfica.

\section{Cinéfilos, Cinépatas}

En Missing, como en Mi cuerpo es una celda, el lazo produce una suerte de quiasmo entre biógrafo y biografiado - biografía y autobiografía - por el que uno y otro resultan intercambiables en el punto en el que literatura y cine se cruzan como tercer factor. El recurso parece proceder también del cine si consideramos que Fuguet elige como epígrafe para Cinépata una de las "Notas para el cinematógrafo" de Robert Bresson en donde el director sostiene que "No se trata de dirigir a alguien sino de dirigirse a uno mismo" (Fuguet 9). La vida del biógrafo — como director de la biografía — se descubre en la curiosidad que lo mueve a introducirse y componer la vida ajena: la posibilidad de contar(se) en el otro. Ante la imposibilidad de significar la vida ajena en su enigma solo queda contarla desde lo que la amistad permite nombrar.

En síntesis, queda claro que, en el caso de las vidas, literaturas y filmografía de Caicedo y Fuguet, el cine supone una experiencia que va más allá de lo que puede nombrar un interés, una práctica del tiempo libre o un objeto de estudio. Podría decirse que es una verdadera "forma de vida"; el estilo de sus biografías; su afección: si Fuguet se autodefine como un "cinépata" además de un "cinéfilo"; Caicedo se decía a sí mismo un "cinesifilítico incondicional” — según lo recuerda su amigo Sandro Romero Rey— en la misma frase de un texto en el que se refiere al autor de Destinitos fatales como un "cinéfago" (Romero Rey 116).

"Amaban el cine porque odiaban la vida, punto" (Fuguet Cinépata 344), sentencia Fuguet en "Cinéfilos", un escrito a medio camino entre el cuento y el ensayo y escrito en la alternancia entre la primera y la tercera persona, que narra una amistad atravesada por la pasión por el cine. La afirmación resume perfectamente las vidas en juego en Mi cuerpo es una celda. Podría decirse que, si en Mi cuerpo es una celda se cuenta la historia de una vida, es de aquella en la que Fuguet y Caicedo pueden por fin coincidir, conocerse, ser amigos y hablar de películas. 


\section{Obras CitAdas}

Andrés Caicedo. 1986. Unos pocos y buenos amigos. Dir. Luis Ospina. Colcultura / Focine. Blanchot, Maurice. 2007. La parte del fuego. Madrid: Arena Libros.

Bourdieu, Pierre. 1997. "Anexo 1. La ilusión biográfica”. Razones prácticas. Sobre la teoría de la acción. Barcelona: Anagrama.

Fuguet, Alberto y Andrés Caicedo. 2008. Mi cuerpo es una celda. Una autobiografía. Santiago de Chile: Norma.

Fuguet, Alberto. 2009. "Prólogo". Caicedo, Andrés. ¡Que viva la música! Bogotá: Norma. . 2010. Missing (Una investigación). Buenos Aires: Alfaguara. . 2012. Cinépata. Una bitácora. Santiago de Chile: Alfaguara.

Holden, Philip. 2014. "Literary biography as a critical form". Biography 37.4 (Fall 2014). Biographical Research Center. Honolulu: University of Hawaii Press.

Lejeune, Philippe. [1975] 1991. "El pacto autobiográfico". Revista Anthropos no 29, Madrid: Anthropos. 47-62

Marcus, Laura. 2002. "The newness of the new biography". Mapping Lives: The uses of biography. France, Peter and William St Clair. Oxford: University Oxford Press.

Musitano, Julia. 2018. "Sobre Sánchez, biografía y abandono". En Un arte vulnerable. La biografía como forma. Avaro, Nora, Julia Musitano, Judith Podlubne Compiladoras). Rosario: Nube Negra.

Pérez, Martín. 2010. "Entrevista a Alberto Fuguet. La película de su vida". Buenos Aires: Radar Libros. Diario Página/12.

Romero Rey, Sandro. 2015. Memorias de una cinefilia (Andrés Caicedo, Carlos Mayolo, Luis Ospina). Bogotá: Universidad del Valle.

Serra Bradford, Matías. 2011. "Prólogo". En Holroyd, Michael. Cómo se escribe una vida. Ensayos sobre biografia, autobiografia y otras aficiones literarias. Buenos Aires: La Bestia Equilátera. 